\title{
Technical Note: Using a high finesse optical resonator to provide a long light path for differential optical absorption spectroscopy: CE-DOAS
}

\author{
J. Meinen ${ }^{1,2}$, J. Thieser ${ }^{2, *}$, U. Platt ${ }^{2}$, and T. Leisner ${ }^{1,2}$ \\ ${ }^{1}$ Institute for Meteorology and Climate Research, Aerosols and Heterogeneous Chemistry in the Atmosphere (IMK-AAF), \\ Karlsruhe Institute of Technology (KIT), Germany \\ ${ }^{2}$ Institut for Environmental Physics (IUP), Atmosphere and Remote Sensing, Ruprecht-Karls-Universität Heidelberg, \\ Germany \\ *now at: Department of Atmospheric Chemistry, Max Planck Institute for Chemistry, Mainz Germany
}

Received: 17 April 2008 - Published in Atmos. Chem. Phys. Discuss.: 4 June 2008

Revised: 23 March 2010 - Accepted: 22 April 2010 - Published: 27 April 2010

\begin{abstract}
Cavity enhanced methods in absorption spectroscopy have seen a considerable increase in popularity during the past decade. Especially Cavity Enhanced Absorption Spectroscopy (CEAS) established itself in atmospheric trace gas detection by providing tens of kilometers of effective light path length using a cavity as short as $1 \mathrm{~m}$. In this paper we report on the construction and testing of a compact and power efficient light emitting diode based broadband Cavity Enhanced Differential Optical Absorption Spectrometer (CE-DOAS) for in situ observation of atmospheric $\mathrm{NO}_{3}$. This device combines the small size of the cavity with the advantages of the DOAS approach in terms of sensitivity, specificity and insensivity to intensity fluctuations of the light source. In particular, no selective removal of the analyte (here $\mathrm{NO}_{3}$ ) is necessary for calibration of the instrument if appropriate corrections are applied to the CEAS theory. Therefore the CE-DOAS technique can - in principle measure any gas detectable by DOAS. We will discuss the advantages of using a light emitting diode (LED) as light source particularly the precautions which have to be considered for the use of LEDs with a broad wavelength range. The instrument was tested in the lab by detecting $\mathrm{NO}_{3}$ formed by mixing of $\mathrm{NO}_{2}$ and $\mathrm{O}_{3}$ in air. It was then compared to other trace gas detection techniques in an intercomparison
\end{abstract}

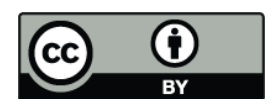

Correspondence to: J. Meinen (jan.meinen@imk.fzk.de) campaign in the atmosphere simulation chamber SAPHIR at Forschungszentrum Jülich at $\mathrm{NO}_{3}$ concentrations as low as $6.3 \mathrm{ppt}$.

\section{Introduction}

Optical absorption spectroscopy is commonly used for trace gas analysis in ambient air since it is a direct, in situ, noninvasive approach to quantify concentrations of gaseous trace species and aerosol. Every absorption spectroscopy methodology makes use of the Lambert-Beer's law:

$I(\lambda)=I_{\text {in }}(\lambda) \cdot \exp [-\sigma(\lambda) \cdot c \cdot L]=I_{\text {in }}(\lambda) \cdot \exp [-\alpha(\lambda) \cdot L]$

Where $I_{\text {in }}(\lambda)$ and $I(\lambda)$ are the light intensity of the source and the intensity after the light has propagated the distance $L$ through an absorbing medium, respectively. The absorbing medium has the absorptivity $\alpha(\lambda)$, which is represented by a concentration $c$ of an absorber with the absorption cross section $\sigma(\lambda)$.

By the application of cavity enhanced light paths in absorption spectroscopy of atmospheric trace gases the detection limits were successfully lowered to sufficient values because the signal increases with the length of the light path $L$. $L_{0}$ is the average length of a light path of a photon inside an empty resonator consisting of two highly reflective mirrors of reflectivity $R$ separated by the distance $d_{0}$. $L_{0}$ has

Published by Copernicus Publications on behalf of the European Geosciences Union. 
to be interpreted as the average light path over all photons or partial rays propagating inside an evacuated resonator before they leave it by passing one of the mirrors (Fielder et al., 2003; Platt et al., 2009). This value can be estimated by:

$L_{0} \approx \frac{d_{0}}{1-R}$

The mirror reflectivity is commonly $R \approx 0.999 \ldots . .0 .999985$ providing a light path $L_{0} \approx 1 \ldots 60 \mathrm{~km}$ suitable for a detection limit of $\alpha \leq 1 \times 10^{-9} \mathrm{~cm}^{-1}$ or below.

There are two basic approaches for cavity enhanced spectrometry relying on a pumped optical resonator: (1) Cavity Ring-Down Spectroscopy (CRDS) measures the decay of light intensity leaking from the resonator after switching off the pump and therefore is a time dependent measurement. (2) Cavity Enhanced Absorption Spectroscopy (CEAS) measures the transmittance of the resonator by recording the leak-out intensity. Both techniques are reviewed e.g. by Ball et al. (2003) and Brown (2003).

In Cavity Ring-Down Spectroscopy, the change in the 1/e decay time of the resonator is measured to determine the absorbance $\alpha$ of a sample. This is an elegant calibration free approach, but the decay time of the empty resonator (which is directly linked to the mirror reflectivity $R$ ) has to be known. So there is the problem of removing the absorber, which might be disadvantageous in field use. One approach is to remove the analyte by titration and to monitor the corresponding change in decay time. The second approach is to use two detection channels: one at the centre of an absorption line and the other at a region of the spectrum where the absorption line is weak. Both approaches are markedly vulnerable to the background extinction and thus only feasible with a very limited number of species of atmospheric interest.

A further improvement was the introduction of Broadband CRDS (BB-CRDS) in 2001 (see review of Ball et al., 2003). This technique provides wavelength resolved absorption spectra, which can be analyzed by fitting routines known from the established Differential Optical Absorption Spectroscopy (DOAS) as introduced by Platt and Perner (1980).

A parallel development was the continuous wave operation of the resonator, first introduced as Cavity Enhanced Absorption Spectroscopy (CEAS) by Engeln et al. (1998). This approach takes advantage of the extension of the absorption light path within the resonator similar to White (White, 1942) or Herriot (Herriot et al., 1965) cells. CEAS uses the integrated leak-out intensity of a beam propagating between two highly reflective mirrors. The absorption coefficient $\alpha$ within the cavity can be related to the leak-out intensity once the amount of light transmitted by the empty resonator and the mirror reflectivity is known. With some basic approximations a simple formula can be derived (Fiedler et al., 2003):

$\alpha=\frac{1}{d_{0}}\left(\frac{I_{0}}{I}-1\right)(1-R)$

Here $I_{0}$ and $I$ are the intensities transmitted through the empty resonator (i.e. filled with pure, synthetic air) and the resonator with an absorbing species inside, respectively. Note that the determination does not rely on the knowledge of the intensity $I_{\text {in }}$ coupled into the resonator, but this method is very sensitive to intensity fluctuations of the light source if they occur between the measurement of $I_{0}$ and $I$. Platt et al. (2009) have recently shown corrections for this approximate formula derived by Fiedler et al. (2003), which are necessary since there is a non-linear dependency between measured intensity and absorber concentration for high concentrations. For broadband techniques it is important to note that all parameters in Eq. (3), except $d_{0}$, are wavelength dependent quantities.

With the development of the incoherent broad-band CEAS (IBB-CEAS) by Fiedler et al. (2003), trace gas analysis with CEAS became feasible. As in BB-CRDS, a wavelength resolved absorption spectrum is obtained from Eq. (3). Column densities of the absorbing trace species are then determined by differential fitting which is the well known DOAS approach (Platt et al., 1980). Langridge et al. (2006) were the first group to point out the advantage of the application of cavity-based techniques allied to the DOAS methodology for in situ measurements using a compact instrument, but corrections for the DOAS data analysis were not applied in their work. For the retrieval of concentrations from column densities the loss factor of the resonator (represented by $R$ in Eq. (3) or the $L_{0}$ in Eq. 2) has to be determined. Broadband devices either rely on separate measurement of the empty cavity or simultaneously determine the absorptions of absorbers with known concentrations (e.g. $\mathrm{H}_{2} \mathrm{O}, \mathrm{O}_{2}$, $\mathrm{O}_{4}$, Platt et al., 1980) as it was introduced in 2004 for DOAS approaches (Hönninger et al., 2004). In the past five years a wide spectrum of derivatives of the IBB-CEAS method has been developed (e.g. BBCEAS, Langridge et al., 2006, SCCEAS, Langridge et al., 2008), W-ICOS, Thompson et al., 2006). They mainly differ in the way of determination of the absolute absorber concentration. In this contribution we use the most direct way to define the light path $L_{0}$ by employing CRD parallel to recording broadband transmission spectra in order to achieve absolute absorber concentrations. The theoretical background of this procedure is discussed in great detail in a corresponding theory paper (Platt et al., 2009).

A complete discussion of the different DOAS approaches in atmospheric science is discussed in (Platt and Stutz, 2008). In comparison to the single wavelength approach particular advantages of the DOAS approach (Platt et al., 1980) include very high sensitivity and selectivity of the measurement. Both properties are owed to the simultaneous recording of the intensity transmitted by the cavity at many different wavelengths, allowing the detection of very weak absorption bands (optical densities of $10^{-3}$ or below). By separating narrow-band "differential" absorption structures due to trace gas molecules from intensity changes which are smoothly varying with wavelength, like changes in mirror reflectivity or Mie scattering from aerosol, the latter effects can be eliminated. The recorded spectra usually encompass several 
absorption bands of the trace species of interest, thus detection becomes very specific and reliable. In fact, even overlapping absorption structures of several species can usually be de-convoluted, which is impossible with the single wavelength approach. Numerous examples of applying this procedure in field measurements are reported in the literature and are summarized in (Platt and Stutz, 2008). As a consequence, no selective removal of the trace gas of interest (by e.g. titration) is required, which allows universal application of our technique in field and laboratory measurements of all trace gases measurable by DOAS, including $\mathrm{NO}_{2}, \mathrm{SO}_{2}$, halogen oxides and aromatics (e.g. Platt et al., 1980; Platt and Stutz, 2008). Moreover, due to the fact that effects of aerosol scattering can be relatively easily removed, measurements in the open air are possible. Here, we confine ourselves to measurements where the aerosol extinction has no significant influence. The corrections which have to be applied for aerosol extinction are described elsewhere (Platt et al., 2009).

We present here an easy to use cavity enhanced spectrometer, especially designed for the detection of $\mathrm{NO}_{3}$ and direct use in combination with classical DOAS instruments. This results in a robust and cost-efficient device for field use comparable to Mini-MAX-DOAS devices (Hönninger, 2004). We build a device capable to detect $\mathrm{NO}_{3}$ in the pptv regime since there is still uncertainty associated with the chemistry of this molecule although it was discovered almost three decades before (Platt et al., 1980; Noxon et al., 1980). For instance heterogeneous reactions of $\mathrm{N}_{2} \mathrm{O}_{5}$ (Brown et al., 2006) influence the atmospheric lifetime of $\mathrm{NO}_{3}$, also $\mathrm{NO}_{3}$ was shown to show strong gradients close to the ground (e.g. Geyer and Stutz, 2004; Brown et al., 2007), moreover $\mathrm{NO}_{3}$ has been found to play a role in nighttime $\mathrm{HO}_{\mathrm{x}}$ radical formation (e.g. Platt, 1990). In order to test our new data analysis approach we took part in an appropriate intercomparison campaign (Dorn et al., 2010). There, our device was compared to other cavity enhanced systems and alternative approaches for trace gas detection and showed good performance compared to established instruments. The scope of our work is to join the relatively new concept of broadband CEAS with the well-established DOAS approach and therewith make the comprehensive expertise developed within the DOAS community available to this technique. There are different ways of evaluating broadband CEAS data, which all have particular drawbacks and advantages. Here we will give the community using DOAS fitting software a tool which allows them to stay with their known systems. For consistency in our nomenclature we call this approach Cavity Enhanced DOAS (CE-DOAS).

\section{Experimental}

The instrument (see Fig. 1) consists of a LED as a light source, a resonator formed by two highly reflective mirrors $\left(M_{1}, M_{2}\right)$, a fiber coupler to collect light leaking through mir- ror $M_{2}$ and either a fiber coupled photomultiplier (PMT) or a fiber coupled spectrograph with a charge coupled device (CCD) camera for time resolved or wavelength dispersed measurements respectively. As detailed in the text below, the light source is driven by a function generator in combination with a HF amplifier or by a constant current source, depending whether time resolved ring-down measurements or wavelength dispersed intensity measurements are desired.

The use of a LED offers considerable advantages with respect to size, complexity and robustness. Previous instruments either suffer from a bulky light source like a pulsed laser source (e.g. Ball et al., 2001), a dye laser system (e.g. Ball and Jones, 2003; Brown, 2003) or a Xe-Arc lamp (e.g. Hamers et al., 2002; Fiedler et al., 2003) or they used inexpensive and small diode lasers. If highly coherent (and hence narrow-band) laser sources are used, large intensity fluctuations can occur because the laser emission profile only overlaps with a few cavity modes. Broad-band light sources, like many conventional laser diodes and light emitting diodes, have short coherence lengths thus avoiding intensity fluctuations, because a large number of cavity modes are excited simultaneously. As some other authors did before (e.g. Ball et al., 2004), we utilized the rapid improvement of the light emitting diode (LED) technology. LEDs are cheap, light weight, and sufficiently stable in emission to be used for absorption spectroscopy. Conveniently, their emission bandwidth is similar to the wavelength range of typical high reflective mirrors. Furthermore the need for mode matching, off-axis alignment or dithering the frequency space of the optical resonator is avoided by the incoherent emission of the LED (e.g. Simpson, 2003; Paul et al., 2001; O'Keefe et al., 1999, respectively). The performance of LED driven instruments is still limited by their surface luminance, even thought it has increased by orders of magnitudes in recent years. Spuler and Linne (2002) gave a comprehensive discussion of the stability of a beam propagating in an optical cavity. For the investigation of the coupling efficiency of light emitted from a LED into an optical resonator, we used ray transfer matrix analysis. Rays of different radial offset and tilt passing the planar outer and the curved inner surface of the mirror and subsequently propagating inside the resonator are calculated for the dimensions of our optical cavity. A periodically repeating path of the rays was typically found for less than 100 round trips inside the cavity. We define an optical path as stable, when the ray is still inside the cavity after 250 round trips and periodicity of the path is observable. By these ray tracing simulations we found, that for stable trapping of a light beam in our optical resonator, its input beam divergence has to be smaller than $0.5 \mathrm{mrad}$. One can easily estimate under these conditions that the LED radiating area actually imaged into the resonator is of the order of $200 \times 200 \mu \mathrm{m}$. This is probably one reason why many authors report a poor coupling efficiency (Ball et al., 2004). It is therefore not essential to use a high total power LED, which gains its power not from high surface luminance but 


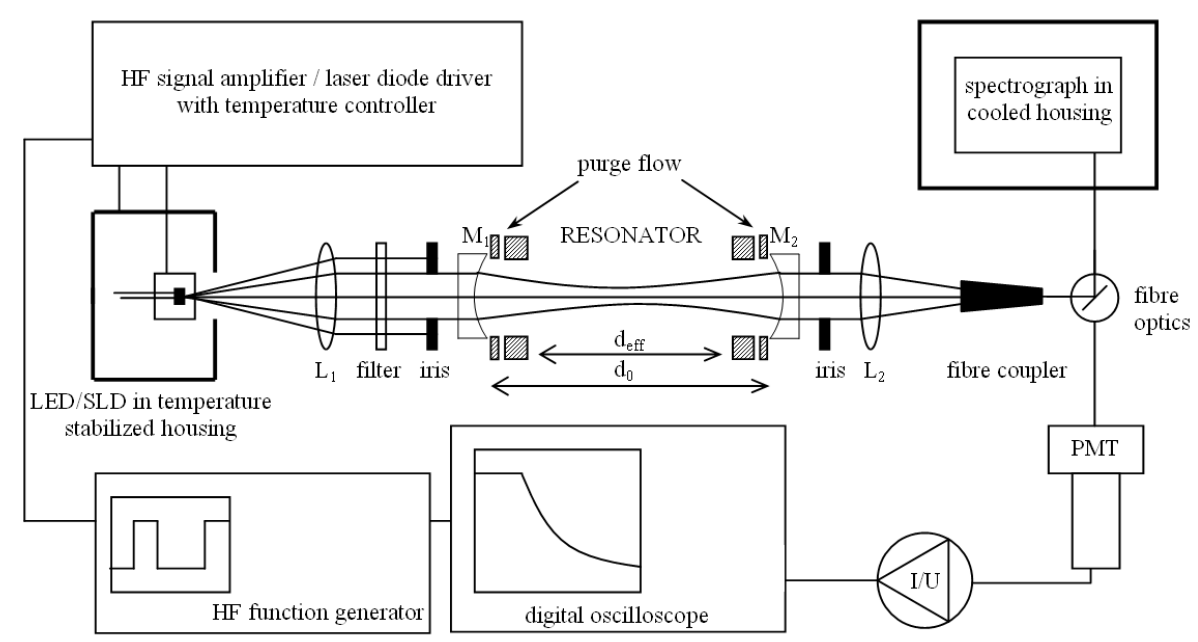

Fig. 1. Schematic diagram of the LED/SLD-based experimental setup used for CE-DOAS. $L_{1}$ and $L_{2}$ : lenses; $M_{1}$ and $M_{2}$ : high reflectivity dielectric mirrors with $R \approx 0.99998$ at $655 \mathrm{~nm}$; Filter: Schott RG610 $3 \mathrm{~mm}$.

rather from a large emitting area. For the wavelength region required, the LED TO3A4-H660-180 (Roithner Lasertechnik $\mathrm{GmbH}$, Austria) was found to perform well. At $25^{\circ} \mathrm{C}$ this LED has sufficient surface luminance of $65 \mathrm{~mW} / \mathrm{mm}^{2}$, its emission spectrum exhibits a nearly Lorentzian shape peaking at $665 \mathrm{~nm}$ with a width of $23 \mathrm{~nm}$ (FWHM) and shows little etalon structure, which is essential for differential fitting.

In this setup, the most effective coupling of the LED light in and out of the resonator was achieved by placing the resonator in between two lens optics $\left(L_{1}=L_{2}: f=40 \mathrm{~mm}\right.$, see Fig. 1). No modification of the LED and no fiber optics are required with this setup. The emission spectrum of the LED covers not only the $662 \mathrm{~nm}$ band, where the measurement is performed, but also the $624 \mathrm{~nm}$ absorption band of $\mathrm{NO}_{3}$ which is vulnerable to photolysis. Therefore a $3 \mathrm{~mm}$ RG610 (Schott, Germany) filter was inserted between the resonator and the light source. The total optical net power at the outer surface of the inlet mirror $M_{1}$ was determined to be about $150 \mu \mathrm{W}$. Thus the reduction of the $\mathrm{NO}_{3}$ concentration in the resonator due to photolysis should be completely negligible.

While all LEDs show a shift in peak wavelength with changing temperature, some LED spectra additionally contain super-imposed periodic structures caused by a FabryPerot etalon effect. The wavelengths at which constructive interference occurs are dependent on the thickness and refractive index of the layers of different materials inside the LED. Because these properties are temperature dependent themselves, the etalon structure changes its spectral position with changing temperature (Kern et al., 2006). A highly stable temperature and emitter current control device was used to minimize shifts in the etalon structure of the LEDs thereby providing a light source well appropriated for differential fitting analysis. For ring-down measurements, a function gen- erator (Model 7060 Generator, Exact Electronics Inc., ORE, USA) was used to trigger a UHF transistor (2N3733) which was interconnected between the current source and the LED. With this setup the edge steepness of the LED light pulse was $<0.8 \mu$ s at a repetition rate of $770 \mathrm{~Hz}$.

The resonator consisted of two high reflective mirrors (Los Gatos Research, Inc., USA) with a nominal reflectivity of $99.9985 \%$ at $655 \mathrm{~nm}, 25.4 \mathrm{~mm}$ diameter and $1 \mathrm{~m}$ radius of curvature. The length $d_{0}$ of the cavity was $620 \mathrm{~mm}$. A fraction of this distance between the mirrors was required for a mirror purge system realized by a counter flow of dry, filtered air (see Fig. 1). It was dimensioned to leave an effective absorption path length $d_{\mathrm{eff}}=(500 \pm 12) \mathrm{mm}$. The characteristic of the flow inside the cavity was optimized by means of computational fluid dynamic (CFD) calculations (ANSYS-CFX, ANSYS, Inc., USA). Typical optical path lengths achieved in the given resonator were between 15 and $20 \mathrm{~km}$. This is a somewhat lower value than expected from the nominal mirror reflectivity, which yields a light path in the region of $40 \mathrm{~km}$. Rayleigh scattering of $\mathrm{N}_{2}$ reduces the maximum possible light path to a value of about $30 \mathrm{~km}$ in this configuration $\left(\sigma_{\mathrm{N}_{2}}(662 \mathrm{~nm}) \sim 2.2 \times 10^{-27} \mathrm{~cm}^{2}\right.$; Sneep et al., 2005). There is still an apparent additional absorbance of about $\alpha=2 \times 10^{-7} \mathrm{~cm}^{-1}$ which indicates degraded mirror surfaces. As the optimal light path for our application is in the region of $12 \mathrm{~km}$ anyhow, (see Sect. 3.2), we did not try to optimize the mirror reflectivity.

The alignment of the resonator was checked with a CCD camera (SensiCam, PCO imaging) equipped with a $25 \mathrm{~mm}$ video lens (Cosmicar/Pentax TV-Lens, $25 \mathrm{~mm}, 1: 1.4$ ). When the image at the output mirror surface was a well defined circle with maximized diameter, transmission was best. This alignment of the lenses corresponds to a focal plane centered between the two mirrors, as described by other authors before 
(Fiedler et al., 2007). The exit beam diameter in the focal plane of $L_{2}$ was measured with the CCD camera to be about $1.6 \mathrm{~mm}$. An optical fiber $(d=400 \mu \mathrm{m}, N A=0.2)$ was placed in the focal spot to guide the light into the detection unit.

For time resolved measurements the cavity output was guided into the PMT (H5783-01, Hamamatsu Photonics Germany $\mathrm{GmbH}$, Hersching) which was operated in continuous current mode. The current was amplified by a fast current to voltage amplifier (DLPCA 200, FEMTO Messtechnik $\mathrm{GmbH}$, Berlin) and digitized by a fast digital oscilloscope (DAQSCOPE PCI-5102, National Instruments Germany $\mathrm{GmbH}$, München). Data recording and processing was managed by LabView software (LabView 8.2, National Instruments Corporation, USA).

Wavelength dispersed measurements were performed with a Czerny Tuner spectrograph (USB 2000, Ocean Optics, Inc., USA) equipped with a CCD array (Sony ILX 511 linear silicon CCD array, 2048 pixels) with a spectral resolution of $1.06 \mathrm{~nm}$ (ca. 6.5 pixel at FWHM) to acquire the spectra. The acquisition rate was limited by exposure and readout time to approximately $1 \mathrm{~min}^{-1}$. Optical bench and detector of the spectrograph were cooled down to approximately $0{ }^{\circ} \mathrm{C}$ in a Mini-DOAS housing (see Hönninger et al., 2004). While taking the spectra, the exposure time was varied automatically to reach constant modulation amplitude for each spectrum. Typically, the exposure time was about $60 \mathrm{~s}$. The spectra are handled and evaluated by using the DOASIS software (Kraus, 2006). For future experiments we plan to enhance the overall performance and temporal resolution using a more sensitive spectrometer with faster readout electronics, more efficient fiber coupling at the exit of the resonator and a more powerful light source (see Sect. 5).

\section{Modification for operation in the SAPHIR chamber}

Since $\mathrm{NO}_{3}$ is a very unstable radical, inlet losses on sample line and cavity housing can potentially cause substantial errors in absolute concentration. Therefore the resonator was placed directly inside the SAPHIR chamber without any tube or housing between the mirrors (open path configuration). This possibility is one of the inherent advantages of the CE-DOAS method. The mirrors were purged by $5 \mathrm{l} / \mathrm{min}$ synthetic air to avoid degradation of the reflectivity during the measurement. A fan placed besides the cavity ensures sufficient circulation of fresh sample air between the mirrors and avoids dilution of the analyzed air due to the purge flow, a prerequisite which presumably would not be necessary in the field due to the always present motion of the air. The LED-light source was directly attached to the resonator inside the chamber but the detection system was kept outside the chamber and was connected to the resonator via a $5 \mathrm{~m}$ long quartz fiber $(D=400 \mu \mathrm{m}, N A=0.22)$.

\section{Data evaluation}

Data evaluation was performed in two steps:

1. Determination of the distance the light travels inside the empty resonator (optical path length $L_{0}$ ). This can be achieved by measuring the attenuation constant of the ring-down signal when the cavity is purged. This procedure is described in detail in Sect. 3.1.

2. Determination of the optical densities $D_{\mathrm{CE}}$ of the different absorbers in the resonator, which is achieved by differential fitting. Absorber concentrations $c$ can be calculated from $L_{0}$ and $D_{\mathrm{CE}}$ including pressure and temperature by the ideal gas law. This procedure is described in Sect. 3.2.

\subsection{Determination of the optical path length $L_{0}$}

For determination of the optical path length the cavity was flushed with dry filtered nitrogen in laboratory use and with synthetic air during an intercomparison campaign detailed below. We assume that finite reflectivity and Rayleigh scattering by nitrogen were the only processes acting to attenuate light within the cavity. 1000 ring-down events were recorded and averaged to reduce noise of the measured intensity decay of the resonator. A mono-exponential function was fitted to this decay (see Fig. 2, dashed line). In Fig. 2 a typical ring-down event with total acquisition time of $1.3 \mathrm{~s}$ is shown. In order to improve statistics of the resulting fit factors, this procedure was repeated 600 times and the fit factors were subsequently averaged for the determination of the optical path length of the empty resonator.

Figure 2 shows the nonlinearity of the logarithm of the ring-down signal due to the summation of varying light paths at different wavelengths covered by the LED emission spectrum. Since the reflectivity of the mirrors peaks at $655 \mathrm{~nm}$ and decreases substantially at the edges of the emission spectrum of the light source, the distance light travels between the mirrors depends considerably on wavelength. This effect becomes even more pronounced, when an absorber with an absorption line narrower than the LED emission spectrum is inside the resonator. In these cases, a simple exponential fit is not suitable for the determination of the light path any more. The time dependent signal $S(t)$ has to be modeled by an integral over the wavelength:

$S(t)=I_{0} \int I(\lambda) \cdot \exp \left[-\frac{t}{\tau(\lambda)}\right] d \lambda+b$

Where $I_{0}$ is the transmitted intensity at $S(t=0)$, and $I(\lambda)$ is the normalized emission spectrum of the LED, $\tau(\lambda)$ is a wavelength dependent time constant given by the wavelength dependent mirror Reflectivity $R(\lambda)$ and $b$ is a baseline (from stray light etc.). This equation can be transformed to:

$S(t)=I_{0} \int I(\lambda) \cdot \exp \left[-\frac{c}{d_{0}}\left(\alpha(\lambda) \cdot d_{\mathrm{eff}}+|\ln R(\lambda)|\right) \cdot t\right] d \lambda+b$ 


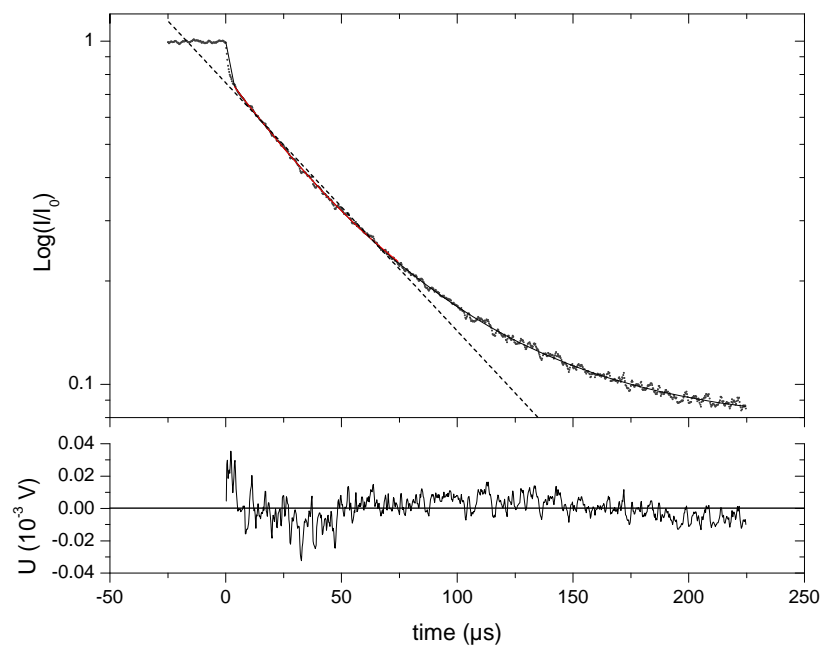

Fig. 2. Ring-down signal averaged from 1000 cycles. Small dots: measurement data. Solid line: simulation result from Eq. (4b). Dashed line: single-exponential fit $(5-75 \mu \mathrm{s})$. Lower panel: fit residual of multi-exponential fit. Note the non-linear decay of $\log \left(I / I_{0}\right)$

Where $c$ is the speed of light, $\alpha(\lambda)$ is the wavelength dependent absorption coefficient, $d_{0}$ and $d_{\text {eff }}$ are the separation of the mirrors and the absorption path length, respectively (see Fig. 1). Note, that this equation accounts for the wavelength dependence of the mirror reflectivity $R(\lambda)$. We performed simulations for an empty resonator $(\alpha(\lambda)=0)$. Equation $(4 \mathrm{~b})$ can be used for the simulation of a ring down event at each wavelength interval based on the appropriate value of $I(\lambda)$ (taken from Fig. 3) but without an absorbing species $\alpha(\lambda)$. The simulation results in a multi exponential decay for a signal integrated over all wavelength. For an integration range from $630 \mathrm{~nm}$ to $690 \mathrm{~nm}$ and an offset $b$ of $10 \%$ representing stray light the signal decay shown in Fig. 2 is well reproduced by the simulation. Since the reflectivity of the mirrors is a priory unknown, we fitted $R(\lambda)$ given by the manufacturer with a constant scaling factor to the data in order to reproduce the decay shown in Fig. 2. The resulting effective reflectivity corrects for resonator losses slowly varying with wavelength (e.g. Rayleigh scattering and mirror degradation; see Platt et al., 2009). We found that fitting a monoexponential function between $5 \mu$ s and $75 \mu$ s to the signal of the empty cavity gives a good estimate for the reflectivity at $660 \mathrm{~nm}$ (see Fig. 2). This method provides a simple and sufficiently accurate way to determine a wavelength independent $L_{0}$ for the use as a path length in the DOAS retrieval with an uncertainty of about $3 \%$. Note that this procedure of calibrating the ring down fit may be different with other mirror - lamp - absorber combinations.

Despite of the fact, that the mean optical path length $L_{0}$ is wavelength dependent in every optical resonator with wavelength dependent mirror reflectivity $R(\lambda)$, for CE-DOAS the

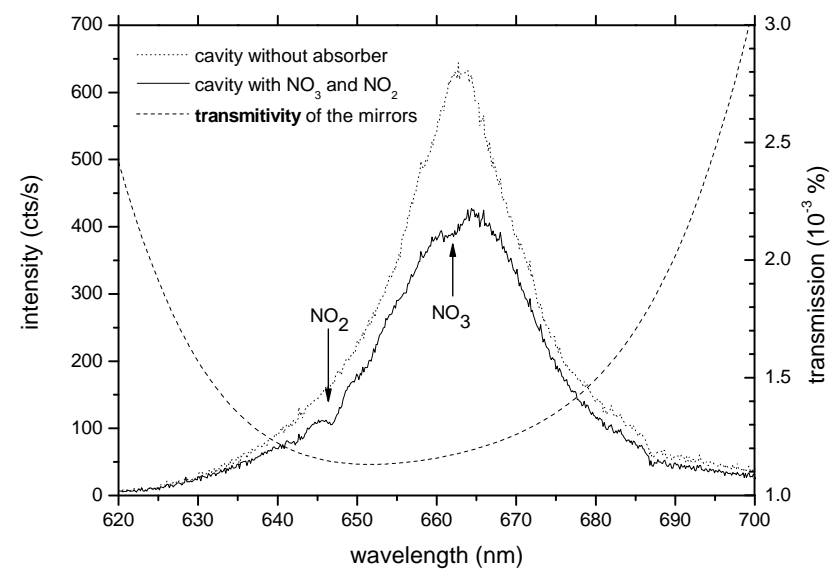

Fig. 3. Transmitted intensity of the resonator: integration time $10 \times 5 \mathrm{~s}$. The dotted line shows the transmitted intensity of the empty cavity, which is the LED emission spectrum folded by the mirror transmission. The solid line was recorded with approx. $0.3 \mathrm{ppbv}$ $\mathrm{NO}_{3}$ and $810 \mathrm{ppbv} \mathrm{NO}_{2}$. The dashed line is the transmissivity of the high reflective mirrors as specified by the manufacturer.

mean optical path length has to be known only at the wavelength were the absorption feature of the target species is strongest. In long path DOAS, a column density is retrieved from the signal. The concentration of the absorbing species can be calculated as the ratio of column density and light path length. Since the reflectivity of the mirrors varies with wavelength, the length of the light path varies accordingly (see Eq. 4b).

\subsection{Differential broadband absorption}

DOAS makes use of Lambert-Beer's law (Eq. 1) to determine the average trace gas concentration $c$. Radiation with the initial intensity $I_{\text {in }}(\lambda)$ is emitted by the source, $I(\lambda)$ is the radiation intensity after passing through a layer of thickness $L$, where the absorber is present. The quantity $\sigma(\lambda)$ denotes the absorption cross section as function of wavelength $\lambda$.

As the radiation propagates through air, its intensity is reduced through the absorption of a specific trace gas. However, it also undergoes extinction due to absorption processes by other trace gases, and scattering by air molecules and aerosol particles. The finite transmissivity of the instrument will also decrease the light intensity. Therefore the simple Lambert-Beer's law (Eq. 1) has to be expanded to consider the various factors that influence the radiation intensity. The absorption of several trace gases with concentration $c_{j}$ and absorption cross sections $\sigma_{j}(\lambda)$, Rayleigh and Mie extinction, $\varepsilon_{R}(\lambda)$ and $\varepsilon_{M}(\lambda)$ and instrumental effects are summarized in $T(\lambda)$ :

$$
\begin{aligned}
& I(\lambda)=I_{\text {in }}(\lambda) \\
& \quad \cdot \exp \left[-L \cdot\left(\sum_{j}\left(\sigma_{j}(\lambda) \cdot c_{j}\right)+\varepsilon_{R}(\lambda)+\varepsilon_{M}(\lambda)\right)\right] \cdot T(\lambda)
\end{aligned}
$$


In order to determine the concentration of a particular trace gas it would be necessary to quantify all other factors influencing the intensity. While previous CEAS and CRDS experiments selectively removed the absorber from the light path this does not appear practical in many cases.

The application of Differential Optical Absorption Spectroscopy overcomes this challenge by using the fact that aerosol extinction processes, instrumental effects, and many trace gas absorptions show very broad or even smooth spectral characteristics. Certain trace gases, however, exhibit narrow band absorptions structures. The foundation of DOAS is thus to separate broad and narrow band spectral structures in an absorption spectrum in order to isolate these narrow trace gas absorptions (Platt et al., 1980). The broad spectrum is then used as a new intensity spectrum $I_{\text {in }}^{\prime}(\lambda)$, and LambertBeer's law can again be applied to the narrow band trace gas absorptions. Accordingly, we split the absorption cross section:

$\sigma_{j}(\lambda)=\sigma_{j 0}(\lambda)+\sigma_{j}^{\prime}(\lambda)$

$\sigma_{j 0}$ in Eq. (6) varies "slowly" with the wavelength $\lambda$, for instance describing a general "slope", such as that caused by Rayleigh and Mie scattering, while $\sigma^{\prime}{ }_{j}$ shows rapid variations with $\lambda$, for instance due to an absorption band. The division between "rapid" and "slow" variations is ambiguous and depends on the observed wavelength interval and the width of the absorption bands to be detected. Inserting Eq. (6) into Eq. (5) we obtain:

$$
\begin{aligned}
& I(\lambda)=I_{\text {in }}(\lambda) \cdot \exp \left[-L \cdot\left(\sum_{j}\left(\sigma_{j}^{\prime}(\lambda) \cdot c_{j}\right)\right)\right] \\
& \cdot \exp \left[-L \cdot\left(\sum_{j}\left(\sigma_{j 0}(\lambda) \cdot c_{j}\right)+\varepsilon_{R}(\lambda)+\varepsilon_{M}(\lambda)\right)\right] \cdot T(\lambda)
\end{aligned}
$$

The first exponential function in Eq. (7) describes the effect of the structured "differential" absorption of a trace species, while the second exponential (including $T(\lambda)$ ) constitutes the slowly varying absorptions, as well as the influence of Rayleigh and Mie scattering (however the influence of broadband absorptions on $L$ does need to be taken into account as we have shown in the corresponding theory paper (Platt et al., 2009). With the quantity $I_{\text {in }}^{\prime}$ as the intensity in the absence of differential absorption

$$
I_{\text {in }}^{\prime}(\lambda)=I_{\text {in }}(\lambda) \cdot \exp \left[-L \cdot\left(\sum_{j}\left(\sigma_{j 0}(\lambda) \cdot c_{j}\right)+\varepsilon_{R}(\lambda)+\varepsilon_{M}(\lambda)\right)\right]
$$$$
\cdot T(\lambda)
$$

we obtain:

$$
\frac{1}{L} \cdot \ln \left[\frac{I_{\text {in }}^{\prime}(\lambda)}{I(\lambda)}\right]=\sum_{j} \sigma_{j}^{\prime}(\lambda) \cdot c_{j}
$$

The intensity $I_{\text {in }}^{\prime}(\lambda)$ is derived by suitable high-pass filtering of the measured intensity $I(\lambda)$. The corresponding differential absorption cross sections $\sigma^{\prime}{ }_{j}(\lambda)$ are determined by applying the same high-pass filter to $\sigma_{j}(\lambda)$, which in turn are measured in the laboratory (i.e. usually taken from the literature). The terms of the left hand side of Eq. (9) are measured, and then the atmospheric trace gas concentrations $c_{j}$ are derived from a least squares fit. A separation of the different absorptions contributing to the sum in equation Eq. (9) is possible because the structures of the trace gases are unique, like a fingerprint.

The practical determination of the optical density was done in the following way: first reference spectra $I_{0}$ were taken in absence of any absorber and scattering particles (i.e. the cavity was flushed with purified air). After that, measurement spectra $I(\lambda)$ were taken with absorber and particles. In order to determine the differential absorption features of the trace gases the logarithm of the quotient of both spectra was taken $\left(\ln \left(I_{0} / I\right)\right)$. Literature reference spectra of the present trace gases were fitted to these differential structures of the measured spectra. The column densities of the fitted reference spectra were determined by a least squares fitting routine. A detailed description of this evaluation procedure is given in Platt and Stutz (2008).

While this formalism works well for long-path (active) DOAS applications, important modifications are required for its application for CE-DOAS. The problem is the dependence of the light path on the trace gas absorption. This can be clarified by considering a situation with mirrors of infinitely good reflectance $(R=1)$ and vanishing extinction by species other than the trace gas to be measured. In this case the loss factor of the cavity and thus the effective light path length $L_{\text {eff }}$ would only be weakly dependant on trace gas concentration $c$ (see Appendix A). We conclude that too high mirror reflectivities (i.e. situations where the losses in the resonator are dominated not by the mirror reflectivity but by the absorption of the trace gas to be measured) can make (sometimes considerable) corrections for the reduction of $L_{0}$ due to trace gas absorptions necessary. The required corrections are described by Eq. (10), the justification of which is detailed in the corresponding theory paper (Platt et al., 2009). Moreover, in order to calculate the optimal mirror reflectivity for a CE-DOAS instrument, the effect of the trace gas absorption, dependent light path length, has to be taken into account.

True $\mathrm{NO}_{3}$ concentrations $\bar{c}$ can be calculated from the optical densities $D_{\mathrm{CE}}$ considering the light path reduction caused by the optical density itself and the uncorrected concentration $c_{0}$ derived from the DOAS fit (see Platt et al., 2009 and Appendix A).

$\bar{c}=c_{0} \frac{e^{D_{\mathrm{CE}}}-1}{D_{\mathrm{CE}}}$

This correction is crucial in all cavity enhanced approaches and in our measurement can amount up to $15 \%$ for $\mathrm{NO}_{3}$ concentrations around 300 pptv. 
The reduction of the light path is not only caused by the target species itself, which can be corrected by Eq. (10), but also by extinction caused by varying concentrations of background aerosol and trace gases with spectrally broad and structure-less absorption. Depending on the measurement principle, there are different approaches to cope with this problem: (1) in single wavelength CRD or CEA one has to remove the aerosol by filtering the analyte and additionally remove all trace gas species by titration, thermal decomposition or photolysis to measure the absolute zero signal. Especially filtering the analyte has the severe drawback that unstable species, e.g. $\mathrm{NO}_{3}$, may be lost. In multi wavelength or broadband approaches, there are three possibilities to solve the problem: (2) filtering of the analyte to achieve a direct measure of the absorption without any scattering. (3) Titration, thermal decomposition or photolysis to remove all trace species for a background which can be subtracted from the signal. (4) Relation of the concentration of the trace species to a known concentration of other absorber e.g. $\mathrm{O}_{2}, \mathrm{O}_{4}$ or $\mathrm{H}_{2} \mathrm{O}$. In this case one can easily determine the aerosol extinction additionally.

\section{Results}

This section presents the results of proof of principle measurements performed on gas phase absorbers in the laboratory and at the intercomparison campaign in the SAPHIR atmosphere simulation chamber. Laboratory measurements on $\mathrm{NO}_{2} / \mathrm{NO}_{3}$ mixtures are shown first to demonstrate the sufficient spectral coverage of the instrument.

\subsection{Laboratory measurements}

$\mathrm{NO}_{3}$ was synthesized in a continuous flow reactor in the reaction sequence $\mathrm{NO}+\mathrm{O}_{3} \rightarrow \mathrm{NO}_{2}+\mathrm{O}_{2}$ and $\mathrm{NO}_{2}+\mathrm{O}_{3} \rightarrow \mathrm{NO}_{3}+\mathrm{O}_{2}$ (Atkinson et al., 2004). In order to achieve a complete $\mathrm{NO}$ to $\mathrm{NO}_{3}$ reaction, we mixed a small fraction of $\mathrm{NO}(\sim 15 \pm 1 \mathrm{ppmv})$ with a large quantity of $\mathrm{O}_{3}$ $(\sim 350 \pm 100$ ppmv $)$ from an electrical discharge ozonizer in a constant flow of dry, filtered air at room temperature. The reaction occurred in a light sealed reaction chamber to avoid decomposition of the $\mathrm{NO}_{3}$ due to photolysis. Reaction chamber and absorption chamber were made of glass and were connected by a $50 \mathrm{~mm}$ long Teflon tube. Tube and the absorption chamber were shielded from ambient light as well. The residence time in the reaction and absorption chamber were $15 \mathrm{~s}$ and $60 \mathrm{~s}$, respectively. In spite of these precautions, $\mathrm{NO}_{2}$ was always detectable in the absorption chamber. A typical CEAS spectrum is shown in Fig. 3. The transmitted intensity with and without an absorber inside the resonator is depicted and compared to the transmission characteristics of the mirrors (data provided by the manufacturer). The $662 \mathrm{~nm}$ absorption band of $\mathrm{NO}_{3}$ is located in the center of the LED emission spectrum and completely in the high reflecting re- gion of the mirrors. Figure 4 shows the feasibility to detect $\mathrm{NO}_{3}$ in the presence of a large background of $\mathrm{O}_{3}$. Concentrations of $(360 \pm 90) \mathrm{ppt} \quad \mathrm{NO}_{3}$ in $(5 \pm 0.9) \mathrm{ppm} \mathrm{O}_{3}$ can be estimated roughly by using Eq. (3) with $L_{0}=11.72 \mathrm{~km}$ and nonlinear least-squares fit to the absorption coefficient:

$a(\lambda)=n_{\mathrm{O}_{3}} \cdot \sigma_{\mathrm{O}_{3}}(\lambda)+n_{\mathrm{NO}_{3}} \cdot \sigma_{\mathrm{NO}_{3}}(\lambda)+T(\lambda)$

In Eq. (11) the number densities $n_{\mathrm{O}_{3}}$ and $n_{\mathrm{NO}_{3}}\left[\mathrm{~cm}^{-3}\right]$ are fit parameters. $\sigma_{\mathrm{O}_{3}}(\lambda)$ and $\sigma_{\mathrm{NO}_{3}}(\lambda)$ are taken from the literature for $T=293 \mathrm{~K}$ and $T=294 \mathrm{~K}$ respectively (UV-VIS Absorption Spectra of Gaseous Molecules and Radicals: http: //www.atmosphere.mpg.de/enid/2295/, accessed: 2 November 2007.), and $T(\lambda)$ is a polynomial representing instrumental effects. The residual is mainly caused by $\mathrm{NO}_{2}$ and water absorption and nonlinear effects of the light source and detection unit.

\subsection{Intercomparison measurements in the SAPHIR chamber}

In order to demonstrate its ability to detect $\mathrm{NO}_{3}$ under more realistic conditions, the instrument took part in an $\mathrm{N}_{2} \mathrm{O}_{5} / \mathrm{NO}_{3}$ intercomparison campaign at the SAPHIR atmosphere simulation chamber at the Forschungszentrum Jülich. The goal of this campaign was to detect sub 100 ppt concentration of $\mathrm{NO}_{3}$ in the presence of water, aerosol and other trace gases.

The CEAS instrument was placed directly inside the chamber to avoid inlet losses. Under these conditions, an effective absorption path length of about $8500 \mathrm{~m}$ was determined by measuring the ring-down signal when the chamber was filled with synthetic air only. The path length was found to vary only slightly from day to day (2.5\% RMS) and therefore was determined prior to each experiment. The estimated uncertainty was $<3 \%$. During the experiment, spectra were acquired with a rate of $1 \mathrm{~min}^{-1}$ by the Mini-DOAS spectrometer described in Sect. 2. The exposure time typically was about $60 \mathrm{~s}$ but was varied automatically to reach constant illumination for all spectra. The data are handled and evaluated by using the software DOASIS (Kraus, 2006). For maximum data reliability, we defined two criteria when the data has to be discarded: first, when the background signal was dominating due to increased scattered light e.g. caused by an opened roof of the chamber. Second, when the fan inside the chamber was turned off, since than the purge flow and wall effects diluted the trace gas concentration between the mirrors in an unpredictable way.

Figure 5 shows raw data recorded from the spectrometer. To obtain low noise background spectra $\left(I_{0}\right)$ a total of 572 spectra were recorded, scaled and averaged overnight (Fig. 5a). During experiment, five subsequent spectra $(I)$ were averaged to maintain sufficient temporal resolution (Fig. 5b). Figure 6 demonstrates how the concentrations are determined by differential fitting to a broadband spectrum smoothed by a low pass filter. The fit shows a good match 


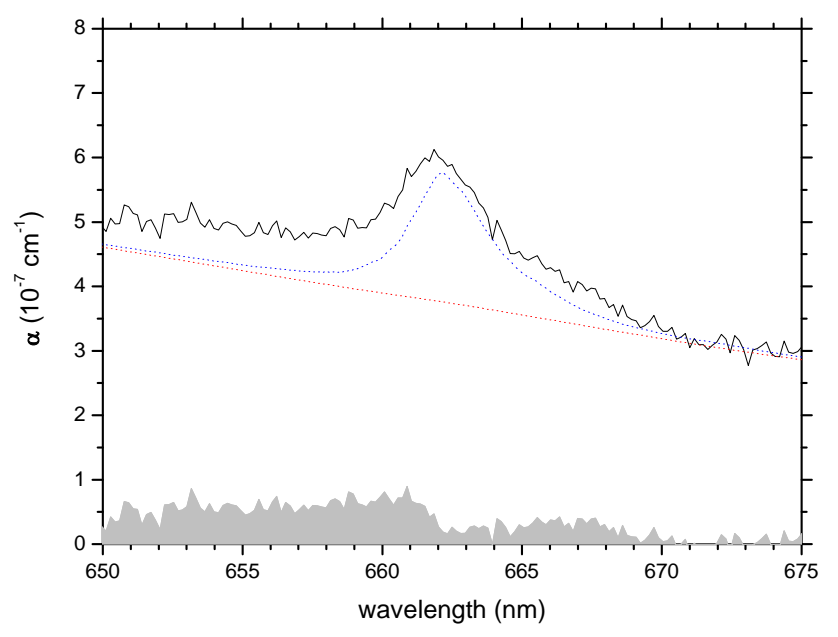

Fig. 4. Detection of $\mathrm{NO}_{3}$ in a large background of $\mathrm{O}_{3}$ in air. The solid line shows the absorption coefficient of the samples calculated by conventional CEAS means from a data set as shown in Fig. 3. The offset is due to absorption of $\sim 5 \mathrm{ppm}$ Ozone in the cavity (dotted, red). The $\mathrm{NO}_{3}$ peak is due to a concentration of $\sim 360 \mathrm{ppt}$ (dotted, blue). Residual (mainly from $\mathrm{NO}_{2}$, grey). This rough estimation of concentration based on Eq. (3) and Eq. (10) coincides with the DOAS analysis results. There was no polynomial used in the fit to avoid co-variation possible with the ozone signal.

to the data in the region of high mirror reflectivity (635$680 \mathrm{~nm})$. The concentration retrieved from this spectrum was $c_{\mathrm{NO}_{3}}=(631 \pm 5)$ pptv. The uncertainty given here is the $1 \sigma$ statistical error associated with the spectral fit. $\mathrm{NO}_{2}$ was not detectable on this experiment day.

Figure 7 shows time series for $\mathrm{NO}_{3}$ concentrations during one experiment day. $\mathrm{NO}_{2}$ and $\mathrm{O}_{3}$ were injected into the light sealed chamber to synthesize a certain amount of $\mathrm{NO}_{3}$. Once the designated concentration was reached, the light shield of the chamber was opened to destroy the $\mathrm{NO}_{3}$ by photolysis (solid bars in Fig. 7). Such photolysis experiments were repeated several times at different concentrations, the corresponding breakdown is clearly visible in our data. Each point in Fig. 7 represents the mean of five spectra recorded. The temporal resolution was given by $5 \times 60 \mathrm{~s}$ for data readout from the CCD plus some seconds for data handling. Therefore the temporal resolution is about $6 \mathrm{~min}$, not sufficient to resolve the dynamics of the single photolysis events in time. The data point marked by an arrow was obtained by analyzing the data shown in Fig. 5 and Fig. 6. The evaluation of the data yields a $2 \sigma$ precision of the CE-DOAS $\mathrm{NO}_{3}$ measurement from 6.3 to $13 \mathrm{pptv}$ at days without high aerosol and high water values (see dotted line in Fig. 7). The $2 \sigma$ precision is represented by two times the fit error.

The good performance of our simple instrument is demonstrated by comparing it to a reference instrument employing a traditional long path DOAS using a white cell (Bossmeyer et al., 2006) at $\mathrm{NO}_{3}$ mixing ratios from about 10 to 600 pptv.

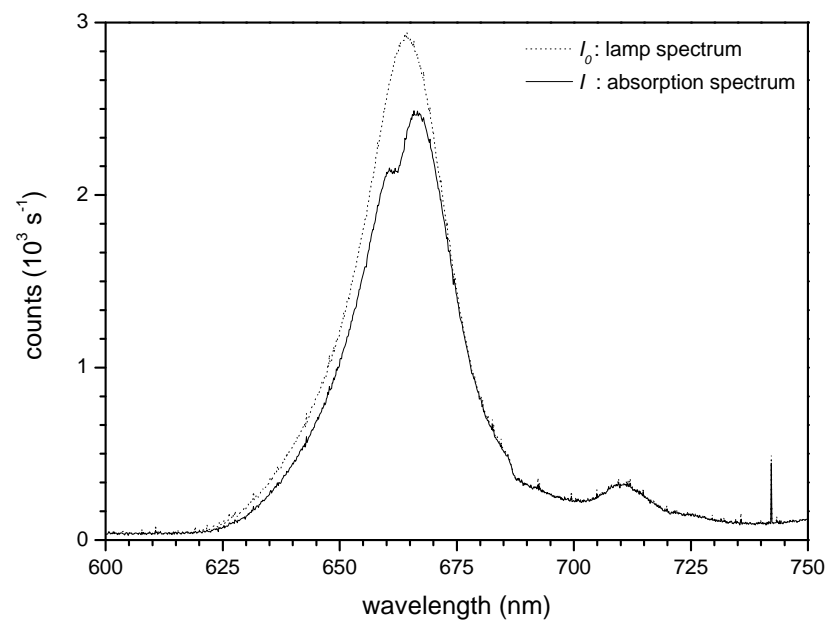

Fig. 5. Recorded CE-DOAS spectra during the $\mathrm{NO}_{3} / \mathrm{N}_{2} \mathrm{O}_{5}$ intercomparison campaign (transmitted intensity of the resonator). (a) $I_{0}$ (lamp) spectra were recorded overnight by averaging 572 scaled spectra (dotted line). (b) During experiments five spectra were averaged (solid line). This absorption spectrum ( $I$ ) shows the attenuation corresponding to 631 pptv $\mathrm{NO}_{3}$.

The correlation between these two instruments was very high with a correlation coefficient $R=0.98$, a constant of proportionality of $1.03 \pm 0.01$ and no offset between the two instruments at the experiment shown in Fig. 7.

The total measurement accuracy is estimated to be better than $15 \%$. This value is made up from the following contributions: the geometrical light path $L_{0}$, which is determined by fitting a simple exponential function to the ring-down signal. By doing so, $L_{0}$ has an uncertainty of smaller than $3 \%$. Furthermore the effective absorption path length $d_{0}$ is determined by the purge flow of each mirror. The fraction of $d$ affected by the purge flow is $6 \mathrm{~cm}$ for each mirror with an uncertainty of about $20 \%$ or $1.2 \mathrm{~cm}$ at each end. This gives an uncertainty of $2.4 \mathrm{~cm}$ of the effective absorption path length between the two mirrors $d_{0}$ which contributes about $5 \%$ to the total uncertainty. The fitting uncertainty in the DOAS evaluation is typically about $5 \%$. Additionally, the uncertainty of the absorption cross section of the species has to be included in the absolute accuracy of the measurement. For the data set used herein, this is about $10 \%$ for $\mathrm{NO}_{3}$ (Yokelson et al., 1994). The correction for the reduced light path (cf. Eq. 11) contributes up to 3\% additional uncertainty for high concentrations of $\mathrm{NO}_{3}(>300 \mathrm{pptv})$. At low concentrations $(<50 \mathrm{pptv})$ this contribution is negligible in the context of these measurements. The uncertainties sum up to a total measurement uncertainty of about $13 \%$. The detection limit of the instrument is given by twice the fit error of the DOASIS software. For experiments with low water vapor and aerosol concentrations a detection limit of about $6-13 \mathrm{pptv}$ (300 s integration) is achieved. 


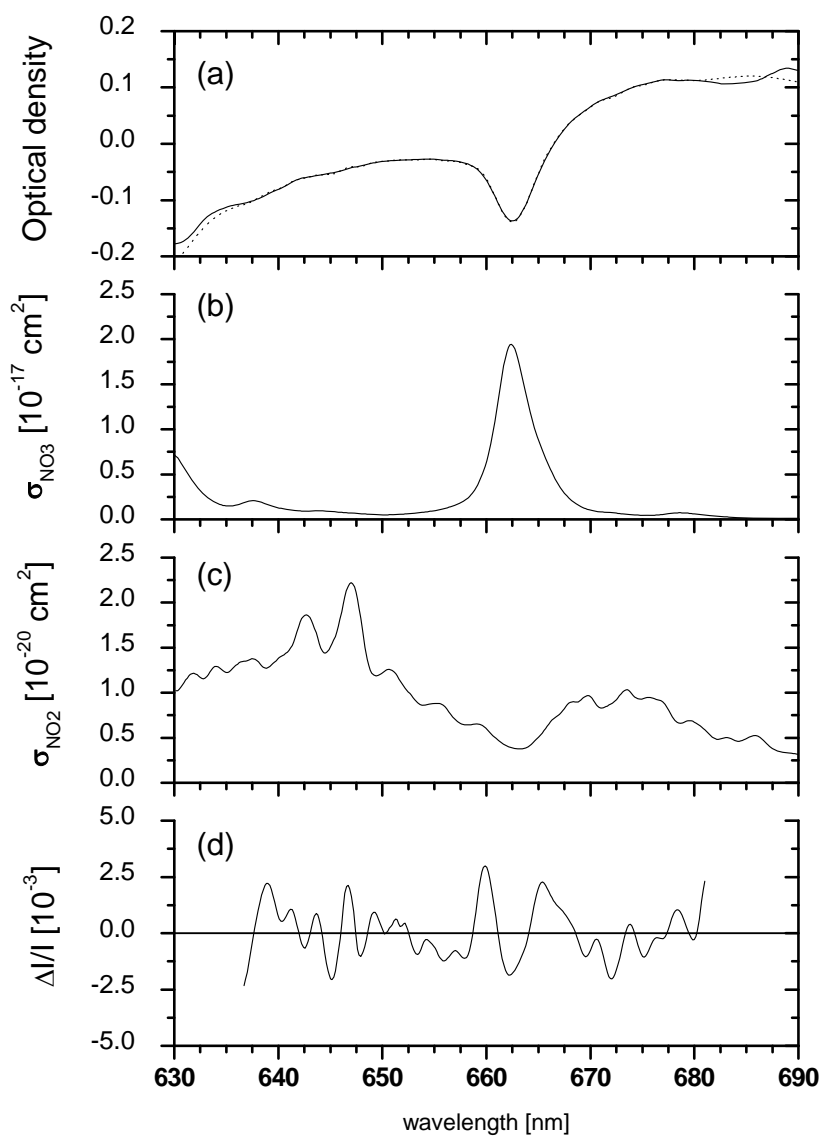

Fig. 6. CE-DOAS spectra of 631 pptv $\mathrm{NO}_{3}$ with traces of $\mathrm{NO}_{2}, \mathrm{O}_{3}$ and $\mathrm{H}_{2} \mathrm{O}$. (a) shows the measured spectrum smoothen with a low pass filter (solid line) and overlaid by the fit result (dotted line). (d) shows the residual spectrum in the data region relevant for the fit. (b) and (c) show the absorption cross sections from $\mathrm{NO}_{3}$ and $\mathrm{NO}_{2}$, respectively.

In the measurements presented here, we operated principally in mode (2) of the possible measurement principles described in Sect. 3.2 (filtering of the analyte to achieve a direct measure of the absorption without any scattering). In the laboratory test case, we synthesized our analyte from gas bottles and sampled it directly into the cavity. Thus no aerosol was able to emerge. During the intercomparison campaign at the SAPHIR chamber the cavity was mounted directly inside the chamber and thus it was accessible to all species which were filled into the chamber for the experiments. In this report, we confined ourselves to the analysis of the data with extreme low or at least low and constant aerosol concentrations. On experiment days with moderate and high aerosol concentrations special corrections have to be concerned in the analysis which are described in detail in the corresponding theory paper (Platt et al., 2009).

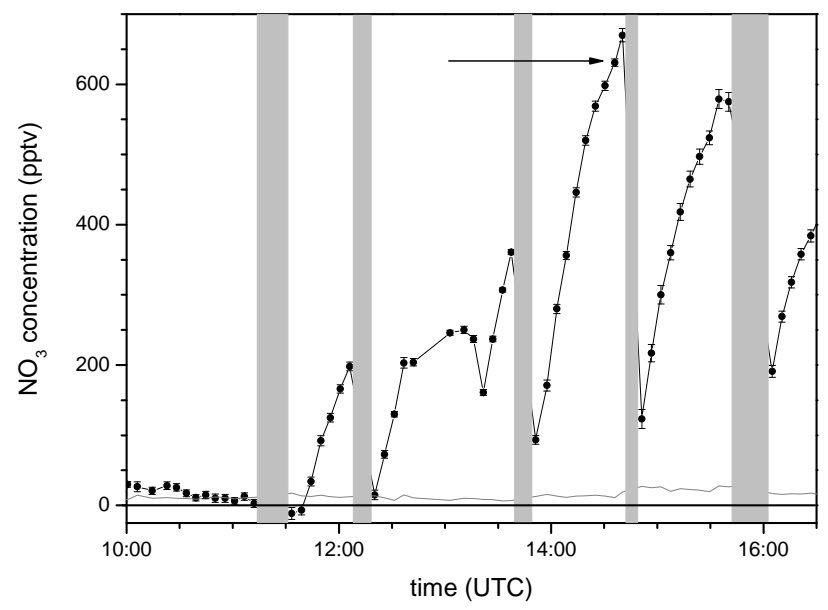

Fig. 7. Time series for $\mathrm{NO}_{3}$ concentration of one experiment day. Each point represents a concentration accessed by DOAS analysis. The grey line near the bottom of the plot denotes the $2 \sigma$ precision of the individual fit result. The data point marked by an arrow was obtained by analyzing the data shown in Fig. 5 and Fig. 6. The solid bars indicate periods were the roof was open and photolysis of $\mathrm{NO}_{3}$ occurred.

\section{Conclusions}

In Cavity Enhanced Differential Optical Absorption Spectroscopy (CE-DOAS) the incoherent radiation of a LED with a relatively broad emission spectrum transmitted by an optical stable cavity is dispersed with a monochromator and detected by a CCD detector. In doing so, this method combines the advantages of sensitivity and specificity of standard non invasive in-situ Differential Optical Absorption Spectroscopy (DOAS) with the enhancement provided by the long light path of an optical resonator. All this is obtained in a very simple and compact setup. In contrast to common BBCEAS techniques, the CE-DOAS method is characterized by its insensitivity to both intensity fluctuations of the light source and the varying background of scattering aerosol. The method employed here is distinguished from other cavity enhanced approaches by accounting for the reduction of the light path by the trace gas absorption. This is essential since cavity losses due to absorption by trace gases reduce the light path length inside the cavity. Reduction of the light path by aerosol extinction was neglected here since these concentration were extreme low or at least low and constant.

The LED light source covers a spectral range of $\Delta \lambda \approx 23 \mathrm{~nm}$ centered at $665 \mathrm{~nm}$, and therefore is applicable for the detection of $\mathrm{NO}_{3}$ with a significant higher spatial resolution than in standard long-path DOAS systems. This can be done without the need of thermal decomposition or titration of the analyte as it is necessary in single wavelength approaches. With total exposure times of $5 \times 60 \mathrm{~s}$ (total acquisition time of $300 \mathrm{~s}$ ), the concentration of $\mathrm{NO}_{3}$ was measured 


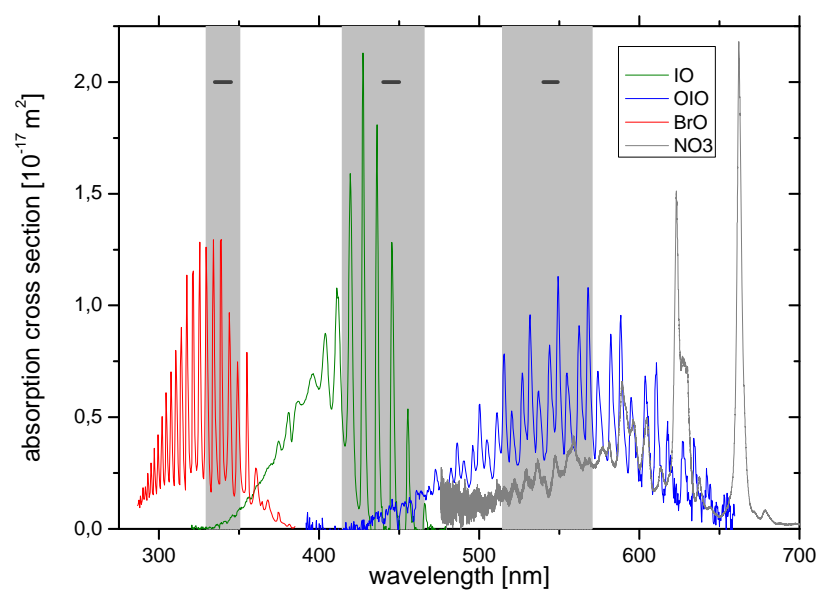

Fig. 8. Absorption cross sections of $\mathrm{BrO}$ (red), IO (green), IOI (blue) and $\mathrm{NO}_{3}$ (dark grey) combined with the spectral regions of high reflective ( $R \sim 0.9998)$ mirrors (light grey bars) and high luminosity LEDs (grey horizontal lines) available from stock. Data from UV-VIS Absorption Spectra of Gaseous Molecules and Radicals: http://www.atmosphere.mpg.de/enid/2295/, accessed: 2 November 2007.

at part per trillion dilution with a statistical uncertainty of \pm 6.3 pptv and an rms noise of $10^{-9} \mathrm{~cm}^{-1}$ in the residual extinction spectrum.

The usability of cavity enhanced techniques is linked to the availability of appropriate mirrors and light sources. Since the coating techniques became state of the art technology in the past years there is virtually no limitation by the availability of mirrors. The need for an appropriate light source has to be considered in more detail but the market is rapidly emerging. So, every species which was detected by DOAS is a promising candidate to be considered for CE-DOAS. To our knowledge there is a nearly continuous availability of LED light sources from $245-700 \mathrm{~nm}$. Only a small fraction of them are availably with very high surface luminosity, but since very long light paths are not needed in every case the available light sources are probably sufficient.

Since atmospheric halogen chemistry (e.g. bromineexplosion) is an interesting field of research, we propose a three color CE-DOAS setup to simultaneously measure IO, $\mathrm{OIO}$ and $\mathrm{BrO}$ in a single measurement. We plan to build a triplex resonator with 340,445 and $545 \mathrm{~nm}$ and to modify our fitting routine in a way, that it includes all three spectra in one fit. Figure 8 shows the absorption cross sections of $\mathrm{BrO}$ (red), IO (green), IOI (blue) and $\mathrm{NO}_{3}$ (grey) combined with the spectral regions of high reflective mirrors and high luminosity LEDs available from stock. The expended detection limit is in the range of the $\mathrm{NO}_{3}$ detection limit reported in this manuscript since the absorption cross sections of the molecules are in the same order of magnitude. LPDOAS measurements in the near UV have shown, that light path $>13 \mathrm{~km}$ are possible without significant limitations due to Rayleigh- and Mie-Scattering (D. Hoch, Diplom thesis, personal communication, 2009; Buxmann et al., 2009).

One of the key limitations of obtaining high temporal resolution at sufficient signal to noise ratios is the amount of light coupled stably in the cavity. Caused by the conservation of radiance only a very small radiating area of the light source can be imaged into the stable region of the resonator. For this reason it is essential to choose a light source with high surface radiance. Some LEDs have promising performance, but they are outperformed by so called Superluminescent Light Emitting Diodes (SLD). These modified laser diodes have a broad $(\Delta \lambda \approx 10 \mathrm{~nm})$ and nearly incoherent emission with a much higher surface luminance than LEDs have. SLDs share all advantages of conventional LEDs except their low cost. Prices of SLDs are comparable to high quality diode lasers. We started test measurements with a $660 \mathrm{~nm}$ SLD and obtained first promising results. Upgrading our CE-DOAS device with a SLD light source will be the scope of a future publication.

\section{Appendix A}

Here we present a short derivation of Eq. (10). For further explanation, please refer to Platt et al., 2009.

In the absence of any extinction in a cavity, half the radiation contained will leave the resonator through mirror 1 , the other half through mirror 2 , each fraction will have the intensity $I_{0}$ :

$I_{0}=I_{L} \cdot \frac{(1-R)}{2}=I_{L} \cdot \frac{\rho}{2}$

The corresponding light path length is:

$\bar{L}_{0}=\frac{d_{0}}{\rho_{0}}$

where $\rho_{0}$ includes the Rayleigh extinction $\varepsilon_{R}$ of pure air in an effective mirror reflectivity

$R_{0}=R-\varepsilon_{R} d_{0}$ or $\rho_{0}=1-R_{0}=-R+\varepsilon_{R} d_{0}=\rho+\varepsilon_{R} d_{0}$.

With additional extinction one can write:

$\bar{L}=\frac{d_{0}}{\rho_{0}+\varepsilon_{B} d_{0}}$

where $\varepsilon_{B}$ denotes all broadband extinctions except Rayleigh scattering.

From the derivative of the intensity inside the cavity $I_{\text {in }}$ one comes to:

$\ln \left(\frac{I_{\text {in }}(0)}{I_{\text {in }}(n)}\right)=n \cdot(1-T R)$

Where $T$ is the transmissivity of the cavity, $R$ is the reflectivity of the mirror and $n$ is the number of light traverses through the cavity. $I_{\text {in }}(n)$ denotes the intensity inside the cavity after 
n reflections. Re-writing $T=1-\tau, R=1-\rho$ with $\tau, \rho \ll 1$ we obtain:

$$
T R=(1-\tau)(1-\rho)=1-\tau-\rho+\underbrace{\tau \rho}_{\approx 0} \approx 1-\tau-\rho
$$

Introducing $T R$ from above in Eq. (A5), and replacing $\rho$ by $\rho_{0}$ (i.e. considering an air-filled cavity, see Eq. A3):

$$
\begin{aligned}
& \ln \left(\frac{I_{\text {in }}(0)}{I_{\text {in }}(n)}\right)=n \cdot(1-T R) \approx n \cdot\left(1-\left(1-\tau-\rho_{0}\right)\right) \\
& \quad=n \cdot\left(\tau+\rho_{0}\right)
\end{aligned}
$$

Note that according to Lambert-Beer's law $\tau$ can be written as:

$$
\tau=\sigma \cdot \bar{c} \cdot d_{0}
$$

Where $\bar{c}$ is the average trace gas concentration in the cavity.

The average number of passes through the cavity results in the decay of the initial intensity inside the cavity $I_{\text {in }}(0)$ to the remaining intensity $I_{\text {in }}(\bar{n})=1 / e \cdot I_{\text {in }}(0)$.

$\ln \left(\frac{I_{\text {in }}(0)}{I_{\text {in }}(\bar{n})}\right)=\ln e=1 \approx \bar{n} \cdot\left(\tau+\rho_{0}\right)$

With the average number of traverses of the photons through the cavity and associated average length of the light path we finally derive for the length of the light path $\bar{L}$ :

$\bar{n}=\frac{1}{\tau+\rho_{0}}$ and $\bar{L}=d_{0} \cdot \bar{n}=\frac{d_{0}}{\tau+\rho_{0}}=\frac{d_{0}}{\sigma \cdot \bar{c} \cdot d_{0}+\rho_{0}}$

Note that in a high finesse resonator a rather weak absorber is already sufficient to significantly reduce the effective light path.

According to DOAS principles (Platt, 1994; Platt and Stutz, 2008) the optical density according to the trace gas to be measured thus becomes:

$D=+\ln \left[\frac{I_{\text {in } 0}(n)}{I_{\text {in }}(n)}\right]=\sigma \cdot \bar{c} \cdot d_{0} \cdot n=\sigma \cdot \bar{c} \cdot L$

Where $I_{\text {in } 0}(n)$ and $I_{\text {in }}(n)$ denote the intensity in the absence and presence of the absorption band, respectively (see Fig. 2 of Platt et al., 2009). From Eq. (A11) the trace gas concentration inside the cavity $\bar{c}$ can be calculated once the absorption cross section $\sigma$ and the light path $L$ is known.

Further consequences of effect of a reduced light path due to absorbers in the cavity are illustrated in the following. The optical density seen by the detector through the second mirror of the cavity, $D_{\mathrm{CE}}$, after $n$ traverses is given by:

$$
\begin{aligned}
D_{\mathrm{CE}} & =\ln \left(\frac{\int_{0}^{\infty} I_{\mathrm{in} 0}(n) d n}{\int_{0}^{\infty} I_{\mathrm{in}}(n) d n}\right)=\ln \left(\frac{\int_{0}^{\infty} I_{\mathrm{in}}(0) \cdot e^{-\rho_{0} n} d n}{\int_{0}^{\infty} I_{\mathrm{in}}(0) \cdot e^{-\left(\rho_{0}+\tau\right) n} d n}\right) \\
& =\ln \left(\frac{\int_{0}^{\infty} e^{-\rho_{0} n} d n}{\int_{0}^{\infty} e^{-\left(\rho_{0}+\tau\right) n} d n}\right)
\end{aligned}
$$

The initial intensity $I_{\text {in }}(0)$ is a (non-zero) constant and can be taken out of the integral in the numerator and denominator. The evaluation of the integral yields:

$$
\begin{aligned}
D_{\mathrm{CE}} & =\ln \left(\frac{\int_{0}^{\infty} e^{-\rho_{0} n} d n}{\int_{0}^{\infty} e^{-\left(\rho_{0}+\tau\right) n} d n}\right)=\ln \left(\frac{-\left.\frac{1}{\rho_{0}} e^{-\rho_{0} n}\right|_{0} ^{\infty}}{-\left.\frac{1}{\rho_{0}+\tau} e^{-\left(\rho_{0}+\tau\right) n}\right|_{0} ^{\infty}}\right) \\
& =\ln \left(\frac{\rho_{0}+\tau}{\rho_{0}}\right)
\end{aligned}
$$

This is the trace gas optical density (or simply the DOAS signal) for a light path $\bar{L}$.

For $\rho_{0} \ll \tau$ the above Eq. (A13) yields $D_{\mathrm{CE}} \approx \ln (\tau)$ being nearly constant. In other words, the trace gas optical density $\mathrm{D}_{\mathrm{CE}}$ will only be weakly dependent on the extinction. For $\rho_{0} \gg \tau$ and constant $\rho_{0}, D_{\mathrm{CE}}$ will be proportional to $\tau=$ $\sigma \cdot \bar{c} \cdot d_{0}$.

In the following we present solutions for the problem of determining the average length of the light path $\bar{L}$ in the presence of absorbers in the cavity. A correction will depend on the mirror reflectivity $R_{0}$ and the extinction in the cavity including the extinction due to the trace gas optical density. Since the true trace gas optical density is a priori unknown, usually an iterative procedure has to be applied.

We have for the trace gas optical density $D_{\mathrm{CE}}$ :

$D_{\mathrm{CE}}=\sigma \cdot \bar{c} \cdot \bar{L}$

From the measured $D_{\mathrm{CE}}$ :

$D_{\mathrm{CE}}=\sigma \cdot \bar{c} \cdot \bar{L} \Rightarrow \bar{L}=\frac{D_{\mathrm{CE}}}{\sigma \cdot \bar{c}}$

Combining Eqs. (A10) and (A15):

$$
\begin{aligned}
\bar{L}= & \frac{d_{0}}{\sigma \cdot \bar{c} \cdot d_{0}+\rho_{0}}=\frac{D_{\mathrm{CE}}}{\sigma \cdot \bar{c}} \text { or }: \sigma \cdot \bar{c} \cdot d_{0}=D_{\mathrm{CE}} \\
& \cdot\left(\sigma \cdot \bar{c} \cdot d_{0}+\rho_{0}\right) \bar{c}\left(\sigma \cdot d-D_{\mathrm{CE}} \cdot \sigma \cdot d\right)=D_{\mathrm{CE}} \cdot \rho_{0}
\end{aligned}
$$

We finally obtain for $\bar{c}$ :

$$
\begin{aligned}
\bar{c} & =\frac{D_{\mathrm{CE}} \cdot \rho_{0}}{\sigma \cdot d_{0}-D_{\mathrm{CE}} \cdot \sigma \cdot d_{0}}=\frac{D_{\mathrm{CE}}}{\sigma} \cdot \frac{\rho_{0}}{d_{0}-D_{\mathrm{CE}} \cdot d_{0}} \\
& =\frac{D_{\mathrm{CE}}}{\sigma} \cdot \frac{\rho_{0}}{d_{0}\left(1-D_{\mathrm{CE}}\right)}
\end{aligned}
$$

Comparing the above expression with the equations for $\bar{c}, \bar{L}_{0}$ as commonly used in DOAS applications we obtain:

$$
\bar{c}=\frac{D_{\mathrm{CE}}}{\sigma \cdot \bar{L}} \text { and } \bar{L}_{0}=\frac{d_{0}}{\rho_{0}}(=\text { path }- \text { length in pure air })
$$

We derive the corrected concentration $\bar{c}$ with absorbers:

$$
\bar{c}=c_{0} \frac{e^{D_{\mathrm{CE}}}-1}{D_{\mathrm{CE}}}
$$


Acknowledgements. The authors appreciate the opportunity to test and compare the instrument during the $\mathrm{NO}_{3}-\mathrm{N}_{2} \mathrm{O}_{5}$ Intercomparison campaign which took place at the atmosphere simulation chamber SAPHIR of the Forschungszentrum Jülich in 2007. This campaign was supported by the European Commision within the 6th Framework Programme, Integrated Infrastructure Initiative: EUROCHAMP (grant no. RII3-CT-2004-505968) and Global Change and Ecosystems: ACCENT. We thank the campaign organizer H.-P. Dorn; T. Brauers and E. Schlosser for providing DOAS data for $\mathrm{NO}_{3}$. The authors thank Denis Pöhler and Holger Sihler for suggestions and advice in terms of the differential fitting utilizing DOASIS.

Edited by: A. Hofzumahaus

\section{References}

Atkinson, R., Baulch, D. L., Cox, R. A., Crowley, J. N., Hampson, R. F., Hynes, R. G., Jenkin, M. E., Rossi, M. J., and Troe, J.: Evaluated kinetic and photochemical data for atmospheric chemistry: Volume $\mathrm{I}-$ gas phase reactions of $\mathrm{O}_{\mathrm{x}}, \mathrm{HO}_{\mathrm{x}}, \mathrm{NO}_{\mathrm{x}}$ and $\mathrm{SO}_{\mathrm{x}}$ species, Atmos. Chem. Phys., 4, 1461-1738, 2004, http://www.atmos-chem-phys.net/4/1461/2004/.

Ball, S. M., Povey, I. M., Norton, E. G., and Jones R. L.: Broadband cavity ringdown spectroscopy of the $\mathrm{NO}_{3}$ radical, Chem. Phys. Lett., 342, 113-120, 2001.

Ball, S. M. and Jones, R. L.: Broad-Band Cavity Ring-Down Spectrscopy, Chem. Rev., 103, 5239-5262, 2003.

Ball, S. M., Langridge, J. M., and Jones, R. L.: Broadband cavity enhanced absorption spectroscopy using light emitting diodes, Chem. Phys. Lett., 398, 68-74, 2004.

Bossmeyer, J., Brauers, T., Richter, C., Rohrer, F., Wegener, R., and Wahner, A.: Simulation chamber studies on the $\mathrm{NO}_{3}$ chemistry of atmospheric aldehydes., Geophys. Res. Lett., 33, L18810, 15, 2006.

Brown, S. S.: Absorption Spectroscopy in High-Finesse Cavities for Atmospheric Studies, Chem. Rev., 103, 5219-5238, 2003.

Brown, S. S., Ryerson, T. B., Wollny, A. G., Brock, C. A., Peltier, R., Sullivan, A. P., Weber, R. J., Dubé, W. P., Trainer, W. P., Meagher, J. F., Fehsenfeld, F. C., and Ravishankara, A. R.: Variability in Nocturnal Nitrogen Oxide Processing and Its Role in Regional Air Quality, Science, 311, 67-70, 2006.

Brown, S. S., Dubé, W. P., Osthoff, H. D., Wolfe, D. E., Angevine, W. M., and Ravishankara, A. R.: High resolution vertical distributions of $\mathrm{NO}_{3}$ and $\mathrm{N} 2 \mathrm{O} 5$ through the nocturnal boundary layer, Atmos. Chem. Phys., 7, 139-149, 2007,

http://www.atmos-chem-phys.net/7/139/2007/.

Dorn, H. P., Apodaca, R. L., Ball, S. M., Brauers, T., Brown, S. S., Crowley, J. N., Dube, W. P., Fuchs, H., Häseler, R., Heitmann, U., Jones, R. L., Labazan, I., Langridge, J., Meinen, J., Platt, U., Pöhler, D., Rohrer, F., Ruth, A. A., Schlosser, E., Schuster, G., Shillings, A., Simpson, W., Thieser, J., Varma, R., Venables, D., and Wahner, A.: Intercomparison of $\mathrm{NO}_{3}$ radical detection instruments in the Atmosphere Simulation Chamber SAPHIR., Atmos. Chem. Phys. Discuss., in preparation, 2010.

Engeln, R., Berden, G., Peeters, R., and Meijer, G.: Cavity enhanced absorption and cavity enhanced magnetic rotation spectroscopy, Rev. Sci. Instrum., 69, 3763-3769, 1998.
Fiedler, S. E., Hese, A., and Ruth, A. A.: Incoherent broad-band cavity-enhanced absorption spectroscopy, Chem. Phys. Lett., 371, 284-294, 2003.

Fiedler, S. E. and Hese, A.: Influence of the cavity parameters on the output intensity in incoherent broadband cavityenhanced absorption spectroscopy, Rev. Sci. Instr., 78, 073104, doi: 10.1063/1.2752608, 2007.

Geyer, A. and Stutz, J.: Vertical profiles of $\mathrm{NO}_{3}, \mathrm{~N}_{2} \mathrm{O}_{5}, \mathrm{O}_{3}$, and $\mathrm{No}_{\mathrm{x}}$ in the nocturnal boundary layer: 2. Model studies on the altitude dependence of composition and chemistry, J. Geophys. Res., 109, D12307, doi:10.1029/2003JD004211, 2004.

Hamers, E., Schram, D., and Engeln, R.: Fourier transform phase shift cavity ring down spectroscopy, Chem. Phys. Lett., 265, 237-243, 2002.

Herriot, D. R. and Schulte, H. J.: Folded Optical Delay Lines, Appl. Optics, 4, 883-889, 1965.

Hönninger, G., von Friedeburg, C., and Platt, U.: Multi axis differential optical absorption spectroscopy (MAX-DOAS), Atmos. Chem. Phys., 4, 231-254, 2004,

http://www.atmos-chem-phys.net/4/231/2004/.

Kern, C., Trick, S., Rippel, B., and Platt, U.: Applicability of lightemitting diodes as light sources for active differential optical absorption spectroscopy measurements, Appl. Optics, 45, 20772088, 2006.

Kraus, S. G.: DOASIS, A Framework Design for DOAS., Doctoral Thesis, University of Heidelberg, Germany, online available at: http://www.iup.uni-heidelberg.de/bugtracker/projects/ doasis/, 2006.

Langridge, J. M., Stephen, M. B., and Jones, R. L.: A compact broadband cavity enhanced absorption spectrometer for detection of atmospheric $\mathrm{NO}_{2}$ using light emitting diodes, Analyst, 131, 916-922, 2006.

Langridge, J. M., Laurilla, T., Watt, R. S., Jones, R. L., Kaminski, C. F., and Hult, J.: Cavity enhanced absorption spectroscopy of multiple trace gas species using a supercontinuum radiation source, Optics Express, 16, 10178-10188, 2008.

Noxon, J. F., Norton, R. B., and Marovich, E.: $\mathrm{NO}_{3}$ in the troposphere, Geophys. Res. Lett., 7, 125-128, 1980.

O'Keefe, A., Scherer, J. J., and Paul, J. B.: cw Integrated cavity output spectroscopy, Chem. Phys. Lett., 307, 343-349, 1999.

Paul, J. B., Lapson, L., and Anderson, J. G.: Ultrasensitive absorption spectroscopy with a high-finesse optical cavity and off-axis alignment, Appl. Optics, 40, 4904-4910, 2001.

Platt, U., Meinen, J., Pöhler, D., and Leisner, T.: Broadband Cavity Enhanced Differential Optical Absorption Spectroscopy (CEDOAS) - applicability and corrections, Atmos. Meas. Tech., 2, 713-723, 2009,

http://www.atmos-meas-tech.net/2/713/2009/.

Platt, U. and Perner, D.: Direct measurements of atmospheric $\mathrm{CH}_{2} \mathrm{O}, \mathrm{HNO}_{2}, \mathrm{O}_{3}, \mathrm{NO}_{2}, \mathrm{SO}_{2}$ by differential optical absorption in the near UV, J. Geophys. Res., 85, 7453-7458, 1980.

Platt, U. and Stutz, J.: Differential Optical Absorption Spectroscopy - Principles and Applications, Series: Physics of Earth and Space Environments, Springer, Berlin, Germany, 597 pp., 2008

Ruth, A. A., Orphal, J., and Fiedler, S. E.: Fourier-transform cavityenhanced absorption spectroscopy using an incoherent broadband light source, Appl. Optics, 46, 3611-3616, 2007.

Simpson, W. R.: Continuous wave cavity ring-down spectroscopy applied to in situ detection of dinitrogen pentoxide $\left(\mathrm{N}_{2} \mathrm{O}_{5}\right)$, Rev. 
Sci. Instr., 74, 1-11, 2003.

Sneep, M. and Ubachs, W.: Direct measurement of the Rayleigh scattering cross section in various gases, J. Quant. Spectrosc. Ra., 92, 293-310, 2005.

Spuler, S. and Linne, M.: Numerical analysis of beam propagation in pulsed cavity ring-down spectroscopy, Appl. Optics, 41, 2858-2868, 2002.

Thompson, J. E. and Spangler, H. D.: Tungsten source integrated cavity output spectroscopy for the determination of ambient atmospheric extinction coefficient, Appl. Phys. B, 91, 195-201, 2006.
Venables, D. S., Gherman, T., Orphal, J., Wenger, J. C., and Ruth, A. A.: High Sensitive in Situ Monitoring of $\mathrm{NO}_{3}$ in an Atmospheric Simulation Chamber Using Incoherent Broadband Cavity-Enhanced Absorption Spectroscopy, Environ. Sci. Technol., 40, 6758-6763, 2006.

White, J. U.: Long optical paths of large aperture, J. Opt. Soc. Am., 32, 285-288, 1942.

Yokelson, R. J., Burkholder, J. B., Fox, R. W., Talukdar, R. K., and Ravishankara, A. R.: Temperature Dependence of the $\mathrm{NO}_{3}$ Absorption Spectrum, J. Phys. Chem., 98, 13144-13150, 1994. 